\title{
Compensation of Bending Waves in an Optically Instrumented Miniature Kolsky Bar
}

\author{
Emily L. Huskins • Daniel T. Casem
}

Received: 9 January 2015/Accepted: 12 January 2015/Published online: 10 February 2015

(C) The authors 2015

\begin{abstract}
Kolsky (split-Hopkinson) bars are susceptible to bending which may introduce flexural wave components to the expected longitudinal loading waves. The traditional method of removing these components by using a pair of strain gages is not practical for bars with very small diameters $(<3 \mathrm{~mm})$. For such bars strain gages are replaced with optical interferometers: a normal displacement interferometer located on the free end of the output bar and transverse displacement interferometer (TDI) together with a diffraction grating on the surface of the input bar. In this work we propose a method to remove bending wave components from the compressive loading pulse through modifications of the existing optical measurement system to include a pair of TDIs that are positioned on opposite sides of the input bar. Short, square longitudinal loading pulses with sub-microsecond rise times, free of bending wave components, were successfully measured using this system.
\end{abstract}

Keywords Kolsky bar · Flexural waves · Particle velocity measurement - Displacement interferometers · High strain rate

\section{Introduction}

The Kolsky (split-Hopkinson) bar [1] is the now classic experiment used to probe the high strain rate $\left(10^{2}-10^{3} \mathrm{~s}^{-1}\right)$

E. L. Huskins $(\bowtie)$

Mechanical Engineering Department, U.S. Naval Academy,

Annapolis, MD 21402, USA

e-mail: huskins@usna.edu

D. T. Casem

U.S. Army Research Laboratory, Aberdeen Proving Ground, MD 21005, USA mechanical response of materials. The diameter of most compression Kolsky bar systems is between 6 and $25 \mathrm{~mm}$. Motivated by the desire to push the boundaries of achievable strain rates to $10^{4}-10^{5} \mathrm{~s}^{-1}$, previous groups have designed miniaturized or 'desktop' Kolsky bar systems with diameters as small as $3-1.5 \mathrm{~mm}$ [2-7]. Regardless of the bar geometry, these systems are susceptible to bending waves due to bar misalignment, asymmetric loading of the specimen, and projectile/input bar misalignment. While the resulting longitudinal and bending waves typically travel at different speeds, they may overlap due to long loading pulses or short bar lengths, creating distortions during data analysis.

For a standard Kolsky bar, the bar strains are recorded with strain gages through Wheatstone bridges. The bending wave effects can easily be removed by mounting a pair of strain gages symmetrically across the bar diameter [8]. This technique cannot be applied with very small diameter $(<3 \mathrm{~mm})$ Kolsky bars, where strain gages cannot be used. The required stress waves are instead recorded using an optical measuring technique proposed by Casem et al. [7]. In this work these optical techniques are further developed to remove bending effects. Experimentation focuses on the presence of bending waves within the incident loading pulse.

\section{Miniature Kolsky Bar}

The miniature Kolsky bar has the same general design as the standard Kolsky bar. Reducing the system geometry aids in obtaining valid high strain rate data in two ways: (1) At higher strain rates the pulse duration is shortened and thus its wave components have shorter wavelengths. These wavelengths must remain significantly larger than the bar 
diameter for the one-dimensional wave propagation assumption to remain valid. Reducing the bar diameter maintains this assumption, preventing significant dispersion effects that greatly reduce the pulse rise time. (2) A state of equilibrium is reached quicker in shorter specimens [9]. This is essential at higher strain rates where significant strains are accrued over short time scales.

The particle displacement in the bar, created by the incident, reflected, and transmitted stress waves, are measured using non-contact, interferometric measurement techniques. These techniques were first applied for pressure-shear plate impact experiments [10] but were recently adapted by Casem et al. for Kolsky bar experiments [6, 7]. Interferometric measurements are made from the interference patterns created by the combination of two waves with the same wavelength. Depending on the phase difference between the waves, either constructive (bright regions) or destructive (dark regions) are observed. As waves go in and out of phase the observed pattern alternates from bright to dark, creating interference fringes. If the path length of one of the waves is dependent on a moveable surface the resulting phase change, measured through the interference fringes, can be related to the displacement of the surface.

Stress waves in the input bar are measured using a transverse displacement interferometer (TDI) utilizing a diffraction grating on the bar surface. Longitudinal displacement of the bar creates interference fringes obtained from the combination of the diffracted beams. ${ }^{1}$ The number of fringes $\left(n_{T D I}\right)$ provides the total longitudinal displacement

$u_{T D I}=\frac{p}{2} n_{T D I}$

where $p$ is the diffraction grating spacing. In addition, a normal displacement interferometer (NDI) is used to measure the longitudinal motion of the free end of the output bar. The number of interference fringes $\left(n_{N D I}\right)$ provides the total displacement of the free end

$u_{N D I}=\frac{\lambda}{2} n_{N D I}$

where $\lambda$ is the wavelength of the light source. Differentiating the displacements in Eqs. 1 and 2 provides corresponding particle velocities respectively

$v_{T D I}=\dot{u}_{T D I} \quad v_{N D I}=\dot{u}_{N D I}$

Note that $v_{N D I}$ is the particle velocity at the free surface. A factor of $1 / 2$ is necessary to describe the particle velocity at other locations within the output bar. Following 1D elastic wave theory one can use these velocities to follow

\footnotetext{
${ }^{1}$ In this discussion it is assumed that the 1 st order diffracted beams are used.
}

the same Kolsky bar data analysis used with traditional strain gage measurements. For a detailed description of these calculations refer to Casem et al. [7].

The miniature Kolsky bar used in this work consists of 17-4 PH stainless steel bars with a diameter of $0.794 \mathrm{~mm}$. Experiments focused on the incident pulse, thus only the input bar was utilized. The projectile is $12.7 \mathrm{~mm}$ long maraging 350 steel and fired from a $0.794 \mathrm{~mm}$ bore gas gun barrel creating a $5 \mu$ s long loading pulse. The bars and barrel rest in a series of precision-made brass bushings that are aligned in a v-notch machined into an aluminum base. Two TDIs are positioned on opposite sides of the input bar (Fig. 1a). The diffraction gratings, $200 \times 300 \mu \mathrm{m}$ (Fig. 2), were milled into the bar using a focused ion beam (FIB) at an accelerated voltage of $30 \mathrm{kV}$ and beam current of $1 \mathrm{nA}$. The line spacing is $1.6 \mu \mathrm{m}$, with individual lines $0.5 \mu \mathrm{m}$ wide and $0.5 \mu \mathrm{m}$ deep. ${ }^{2}$ The gratings are located at the mid-point of the input bar and centered on $300 \mu \mathrm{m}$ wide polished flats which extend the entire length if the bar, maintaining a constant bar cross-section. The light source was a $150 \mathrm{~mW}, 352 \mathrm{~nm}$ wavelength laser. Interference fringes were collected using Thorlabs PDA10A silicon amplified detectors with $150 \mathrm{MHz}$ bandwidth.

The TDI system requires alignment of the laser such that the incident beam is (a) perpendicular to the bar axis and (b) perpendicular to the polished flats. Uncertainty due to misalignment of the former was discussed in Kim et al. 1977 [10] who found that error due to transverse displacement is negligible for sufficiently small angles. Good TDI system alignment was confirmed when the 0th order diffracted beam overlaps the incident beam as observed for a $\sim 5 \mathrm{~mm}$ diameter spot size at a distance of $\sim 1 \mathrm{~m}$. To improve the quality of the interference fringes the optics were positioned such that all incident, diffracted, and combined beams remained collimated and relative path lengths were the same.

\section{Bar Motion due to Flexural and Longitudinal Waves}

A schematic of the incident bar is shown in Fig. 3. The coordinate field is oriented such that the beam's axis is along $\hat{e}_{1}$ and its cross-section is parallel to the $\hat{e}_{2}-\hat{e}_{3}$ plane. The locations of the diffraction gratings, points $\mathrm{C}$ and $\mathrm{D}$, are located in the $\hat{e}_{1}-\hat{e}_{2}$ plane. These points are displaced to $\mathrm{C}^{\prime}$ and $\mathrm{D}^{\prime}$ as the bar deforms due to both flexural and longitudinal waves.

\footnotetext{
${ }^{2}$ Groove geometry selected such that depth $\sim \lambda$ ensuring that the 0th order diffraction was observable.

${ }^{3}$ Experiments conducted at power of $30 \mathrm{~mW}$ provided sufficient contrast of interference fringes. Further optimization of fringe geometry may increase diffraction efficiency, reducing the laser power required.
} 
Fig. 1 a A dual TDI

measurement system, positioned on opposite sides of the bar, used to compensate for bending waves and $\mathbf{b}$ A combined NDI and TDI system, used to measure the lateral displacement of the bar due (primarily) to flexural waves. Diffraction gratings on the bars are not drawn to scale
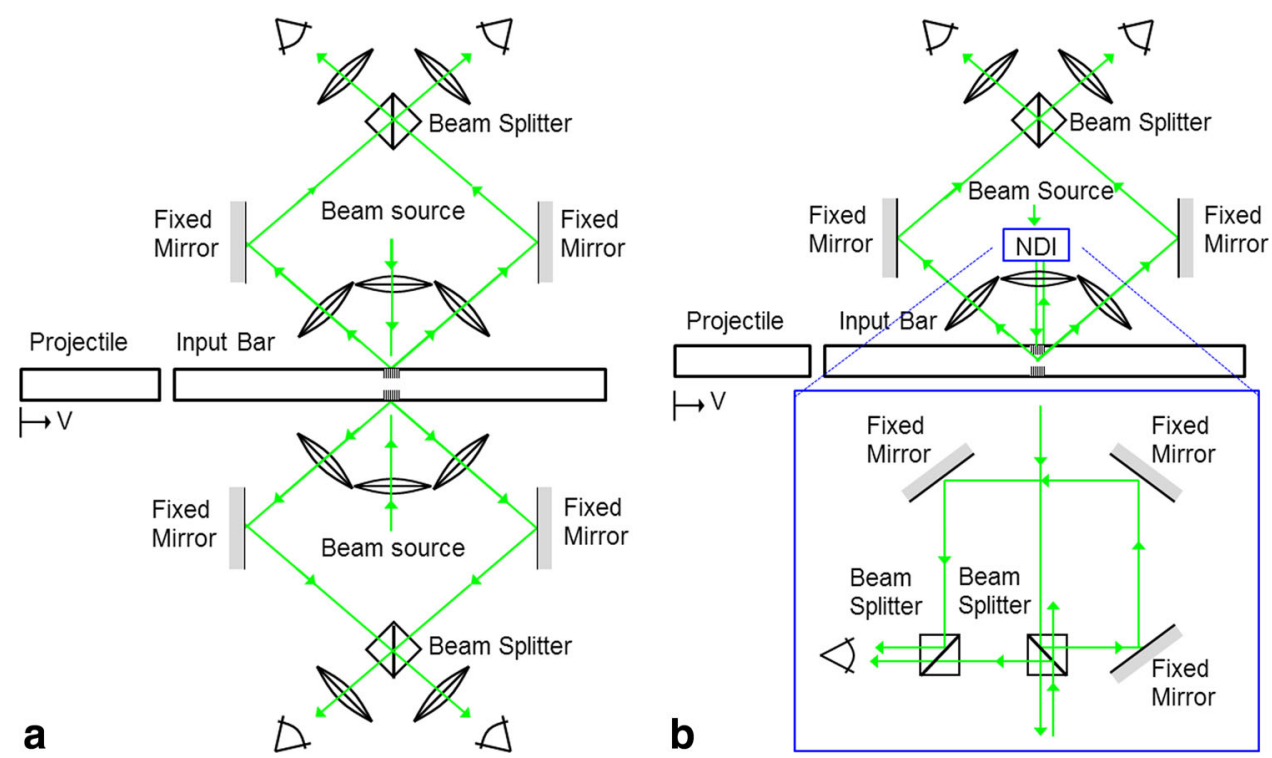
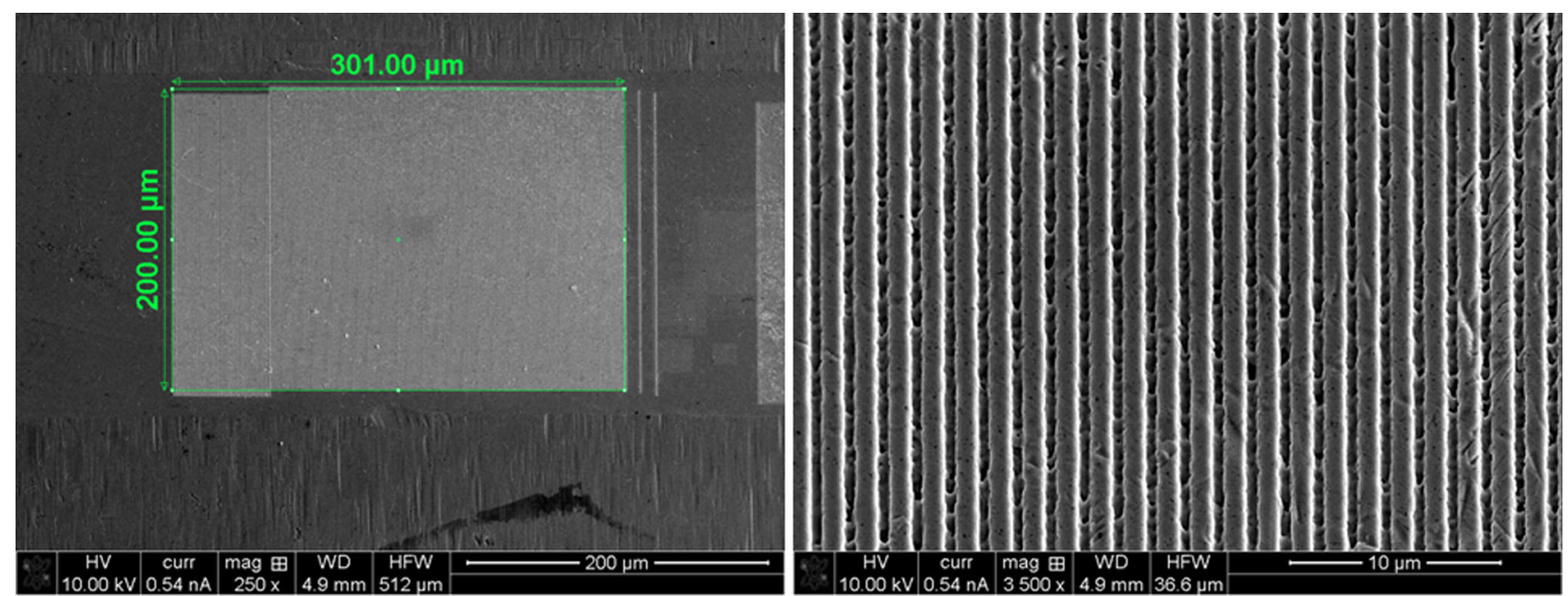

Fig. 2 Micrograph of the diffraction grating milled on to the input bar with a FIB. The line spacing is $1.6 \mu \mathrm{m}$

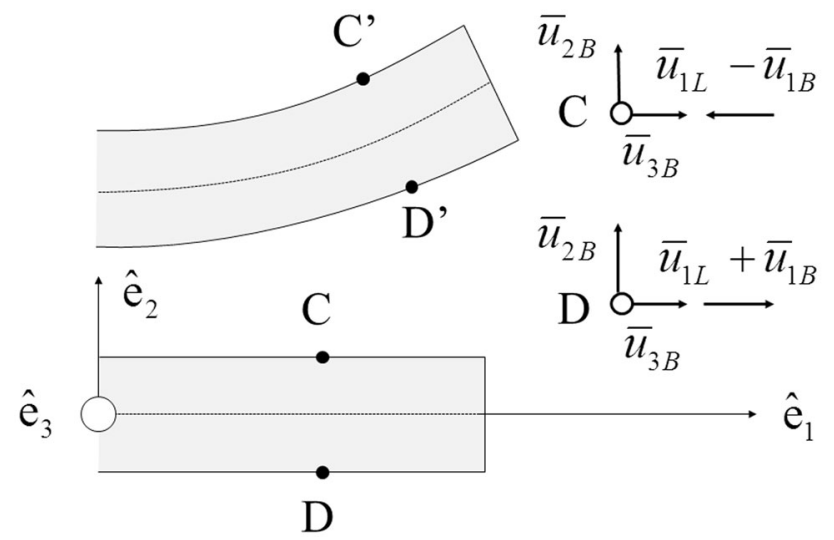

Fig. 3 Schematic of the input bar motion due to flexural and longitudinal waves. Points $\mathrm{C}$ and $\mathrm{D}$ represent the positions of the diffraction gratings
The displacement components at both positions $\mathrm{C}$ and $\mathrm{D}$ are also shown in Fig. 3. Following 1D wave theory, a longitudinal wave will result in only axial displacement, $\bar{u}_{1 L} \hat{e}_{1}$, that is uniform across the cross-section [1]. In this case lateral motion due to the Poisson effect is assumed small in comparison to the longitudinal motion, and for a typical pulse used here is only about $0.5 \%$. The presence of flexural waves within the beam will result in additional displacement components $[11,12]$. In Fig. 3 , the orientation of the bending moment due to the flexural wave is arbitrary with respect to the selected coordinate system, therefore it is decomposed into portions along $\hat{e}_{3}$ and $\hat{e}_{2}$. Torsion associated with rotations about $\hat{e}_{1}$ is ignored (and would not be expected from our loading). Following EulerBernoulli beam theory, bending about $\hat{e}_{3}$ will result in two displacement components at $\mathrm{C}$ and $\mathrm{D}$ : a transverse 
displacement $\bar{u}_{2 B} \hat{e}_{2}$ due to rigid body translation and an axial displacement $\bar{u}_{1 B} \hat{e}_{1}$ due to rigid body rotation. The axial displacements will have equal magnitude but opposite directions (i.e. extension and compression). Bending about $\hat{e}_{2}$ will result in only a transverse displacement, $\bar{u}_{3 B} \hat{e}_{3}$, due to rigid body translation at $\mathrm{C}$ and $\mathrm{D}$. While an axial displacement due to rigid body rotation is produced by bending about $\hat{e}_{2}$, its magnitude at points $\mathrm{C}$ and $\mathrm{D}$ is zero. Note, a similar approach to correcting for bending waves was applied using PDV instrumentation of larger diameter bars used for wave separation [13].

\section{Bending Waves in a Miniature Kolsky Bar}

Figure 4 shows the particle velocity history of the incident pulse recorded by one of the TDIs. Here positive velocity is considered compressive. The pulse has a $0.5 \mu$ s rise time and is approximately square except for strain oscillations in the latter half. Also plotted is the detector output for an NDI constructed with the zero-order beam line within the TDI [10] (Fig. 1). Both data were therefore obtained from the same position on the bar and are correlated in time. Following the coordinate system in Fig. 3, the NDI is only sensitive to transverse displacements along $\hat{e}_{2}$ while the TDI is only sensitive to axial displacements along $\hat{e}_{1}$ [10]. Any displacements along $\hat{e}_{3}$ are therefore not recorded with this system. Note an NDI would not normally be used in this configuration during a Kolsky bar experiment. It is used here specifically to measure the lateral motion experienced by the grating.

Two distinct fringe signals are obtained by the NDI. The first occurs at the arrival of the longitudinal compression pulse and is believed to be the Poisson effect. The second

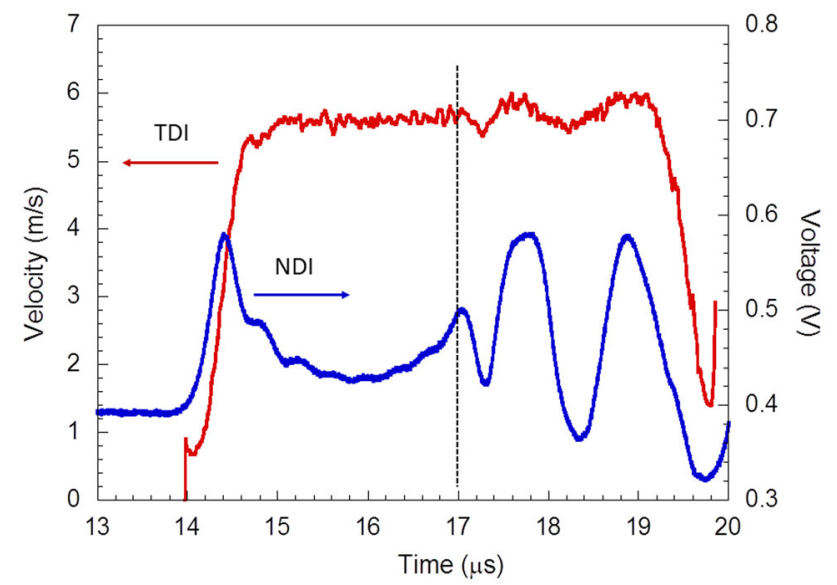

Fig. 4 Particle velocity history obtained from TDI and NDI interference fringes obtained at the same bar location. NDI fringes are observed at the arrival of the longitudinal loading pulse and when strain oscillations occur (dashed line) correlates with the onset of the strain pulse oscillations (dashed line in Fig. 4). In the presence of only longitudinal loading one would expect $u_{N D I}=0$ and $u_{T D I}=\bar{u}_{1 L}$. Deviation from square pulse behavior in addition to the measured transverse displacement suggests additional loading waves are present. Flexural loading leads to complex motions resulting in oscillating displacements, both axial and transverse. This is consistent with the axial strain oscillations in Fig. 4. Unfortunately, transverse displacement oscillations cannot be determined as the NDI cannot unambiguously distinguish changes in the direction of motion.

\section{The Dual TDI System}

Single TDI data, such as that in Fig. 4, cannot be used alone to separate the axial displacement components due to flexural and longitudinal waves. Instead, two TDIs, arranged as in Fig. 1b, can either isolate the axial displacement due to bending,

$\bar{u}_{1 B}=\frac{1}{2}\left(u_{T D I}^{D}-u_{T D I}^{C}\right)=\frac{1}{2}\left(\left(\bar{u}_{1 L}+\bar{u}_{1 B}\right)-\left(\bar{u}_{1 L}-\bar{u}_{1 B}\right)\right)$.

Or alternately, the bending components can be removed, leaving only the axial displacement due to the longitudinal compressive wave

$\bar{u}_{1 L}=\frac{1}{2}\left(u_{T D I}^{D}+u_{T D I}^{C}\right)=\frac{1}{2}\left(\left(\bar{u}_{1 L}+\bar{u}_{1 B}\right)+\left(\bar{u}_{1 L}-\bar{u}_{1 B}\right)\right)$.

From experiments conducted using dual TDIs the axial displacements due to bending rotation, calculated from Eq. 4, were very small, only $0.3 \mu \mathrm{m}$. By comparison, the total axial displacement was $35 \mu \mathrm{m}$. Due to the small difference in displacements it is easier to consider bar velocity, as defined by Eq. 3.

Figure 5 presents the axial velocity histories of two experiments as recorded by each TDI $\left(\mathrm{TDI}_{\mathrm{C}}\right.$ and $\mathrm{TDI}_{\mathrm{D}}$ representing their respective positions at $\mathrm{C}$ and $\mathrm{D}$ in Fig. 3). In Fig. 5a high frequency oscillations are observed following loading. These oscillations are due to dispersion of the high frequency components present in the rectangular loading pulse [8] and are identically observed in both $\mathrm{TDI}_{\mathrm{C}}$ and $\mathrm{TDI}_{\mathrm{D}}$. In Fig. 5b the pulse was shaped slightly to remove the higher frequency components resulting in the relatively flat pulse following loading as observed by both $\mathrm{TDI}_{\mathrm{C}}$ and $\mathrm{TDI}_{\mathrm{D}}$. These experiments confirms that the dual TDI system is capable of capturing the effects of dispersion (or lack thereof) analogous to what is recorded with a pair of foil strain gages used on a traditional Kolsky bar systems [8].

In the latter half of the loading pulses in Fig. 5 the particle velocities recorded by $\mathrm{TDI}_{\mathrm{C}}$ and $\mathrm{TDI}_{\mathrm{D}}$ show equal 

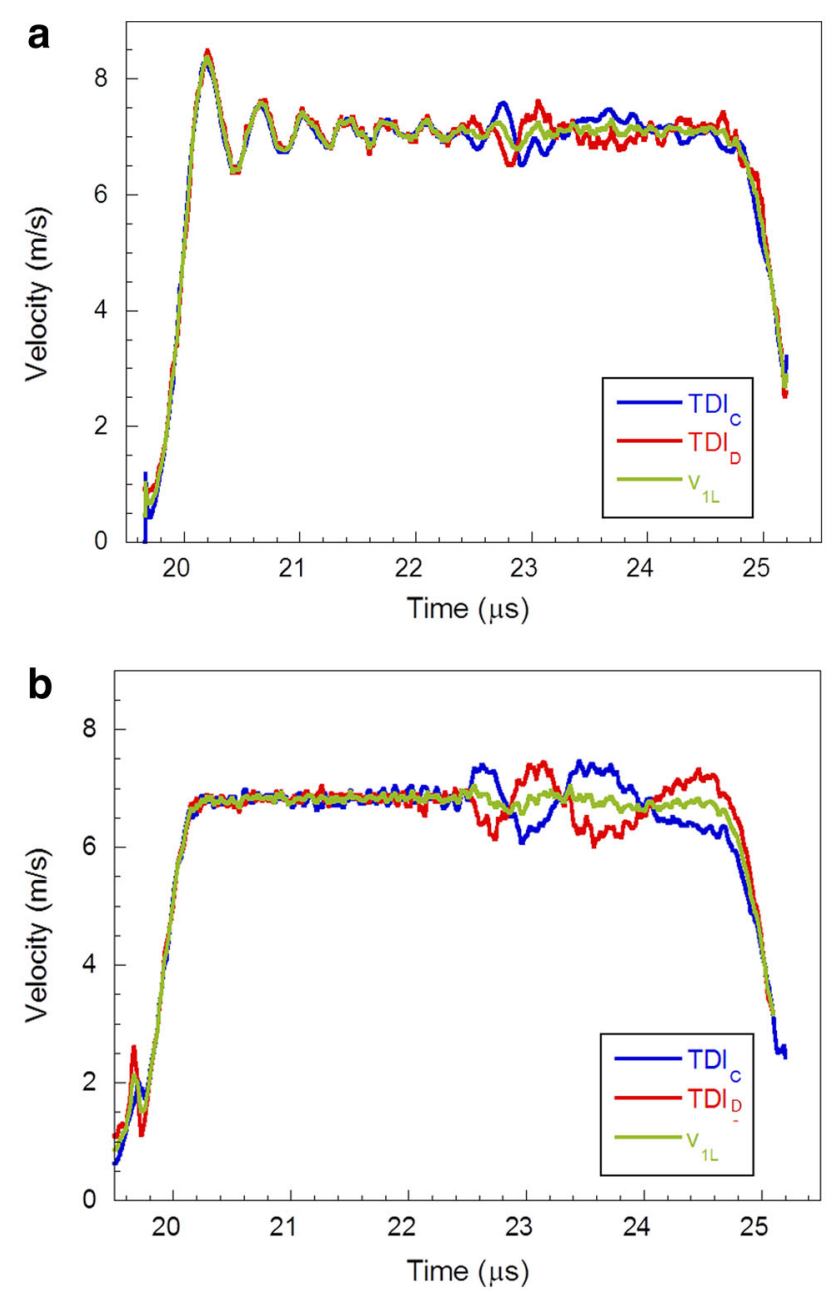

Fig. 5 Bar velocity histories from two TDIs plotted with the corrected axial velocity history associated with the longitudinal loading pulse: $\mathbf{a}$ with dispersion effects and $\mathbf{b}$ in the absence of dispersion effects

but opposite oscillations which are independent of the presence of dispersion. The onset of this deviation corresponds with the onset of bending waves shown in Fig. 4. To determine if these oscillations are, in fact, a result of bending, the axial velocities due to only the longitudinal wave, as calculated from the time derivative of Eq. 5, are also plotted in Fig. 5 (labeled as v). As expected, the dispersion effects remain unaltered. The latter oscillations, however, are nearly entirely removed, leaving a rectangular shaped pulse. This confirms that these oscillations were a result of bending waves and are not present in the longitudinal loading pulse.

\section{Conclusions}

A miniature Kolsky bar with bar diameter of 794 microns was used to create a compressive loading pulse having $5 \mu$ s duration and $0.5 \mu \mathrm{s}$ rise time. Flexure waves due to bending of the incident bars were identified in addition to the expected longitudinal waves. It was found that the components of the incident pulse associated with bending could be removed using a dual TDI system, leaving only the axial particle velocity data resulting from the uniaxial compressive loading pulse. During a Kolsky bar experiment this technique can in principle also be applied to the reflected pulse as well. The use of an NDI on the output bar, however, requires additional considerations to remove bending wave effects.

Acknowledgments This study was supported by the US Army Research Laboratory through the Oak Ridge Institute for Space and Education (ORISE) program \#1120-1120-99.

\section{References}

1. Kolsky H (1949) An investigation of the mechanical properties of materials at very high rates of loading. Proc Phys Soc Lond Sect B 62(11):676

2. Kamler F, Niessen P, Pick RJ (1995) Measurement of the behaviour of high-purity copper at very high rates of strain. Can J Phys 73(5-6):295-303. doi:10.1139/p95-041

3. Jordan JL, Siviour CR, Foley JR, Brown EN (2007) Compressive properties of extruded PTFE. Polymer 48(14):4184-4195. doi:10. 1016/j.polymer.2007.05.038

4. Gorham DA, Pope PH, Field JE (1992) An improved method for compressive stress-strain measurements at very high strain rates. Proc R Soc Lond A 438(1902):153-170. doi:10.1098/rspa.1992. 0099

5. Jia D, Ramesh KT (2004) A rigorous assessment of the benefits of miniaturization in the Kolsky bar system. Exp Mech 44(5):445454. doi:10.1007/bf02427955

6. Casem D, Grunschel S, Schuster B (2011) Interferometric measurement techniques for small diameter Kolsky bars. In: Proulx T (ed) Dynamic behavior of materials, volume 1. Conference Proceedings of the Society for Experimental Mechanics Series. Springer, New York, pp 463-470. doi:10.1007/978-1-4419-82285_70

7. Casem DT, Grunschel SE, Schuster BE (2012) Normal and transverse displacement interferometers applied to small diameter Kolsky bars. Exp Mech 52(2):173-184. doi:10.1007/s11340-0119524-x

8. Chen WSB (2011) Split Hopkinson (Kolsky) bar. Mechanical engineering series. Springer, New York

9. Davies EDH, Hunter SC (1963) The dynamic compression testing of solids by the method of the split Hopkinson pressure bar. J Mech Phys Solids 11(3):155-179. doi:10.1016/0022-5096 (63) $90050-4$

10. Kim KS, Clifton RJ, Kumar P (1977) A combined normal- and transverse-displacement interferometer with an application to impact of y-cut quartz. J Appl Phys 48(10):4132-4139. doi:10. $1063 / 1.323448$

11. Graff KF (1991) Wave motions in elastic solids. Dover, New York

12. Bauchau OA, Craig JI (2009) Structural analysis: with applications for aerospace structures. Springer

13. Casem DT, Zellner MB (2013) Kolsky bar wave separation using a photon doppler velocimeter. Exp Mech 53(8):1467-1473. doi:10.1007/s11340-013-9735-4 